\title{
Hermes: um modelo para acessibilidade ubíqua dedicado à deficiência auditiva
}

\author{
Carlos Felipe Rocha Carneiro ${ }^{1}$ \\ Jorge Luís Victória Barbosa ${ }^{2}$
}

\begin{abstract}
Resumo: Nota-se, atualmente, que o avanço da tecnologia e a crescente quantidade de dispositivos móveis vêm estimulando o uso desse tipo de tecnologia. Porém, esses dispositivos não estão prontos para atender pessoas com determinados tipos de deficiência, especificamente o deficiente auditivo. $\mathrm{O}$ presente trabalho tem por objetivo propor um modelo de suporte ao deficiente auditivo chamado Hermes. O Hermes no que diz respeito aos trabalhos relacionados é o único a apresentar sensibilidade ao contexto, pois nenhum dos modelos avaliados tem essa característica. Além disso, Hermes suporta o reconhecimento de som, a localização de recurso e, por fim, demonstra suporte a trilhas. A avaliação do modelo foi baseada em cenários, partindo de um recurso selecionado pelo usuário, mostrando que o aplicativo oferece suporte para acessibilidade. O Hermes foi avaliado por dez usuários, um deles deficiente auditivo. Os avaliadores aprovaram com $88 \%$ o aplicativo no quesito que buscou avaliar a facilidade percebida de uso, e com $90 \%$ o quesito de utilidade percebida.
\end{abstract}

Palavras-chave: Acessibilidade Ubíqua, Computação Ubíqua, Deficiência Auditiva.

\begin{abstract}
It is perceivable that the technology advance and the increase on the amount of mobile devices have stimulated the use of the mobile technology. Nevertheless, those kinds of device are not completely available to people who have some types of disability, especially the hearing disability. This paper aims to propose a sample of support to deaf people, an application called Hermes. Hermes is different from the other applications because it is sensitive to the context. Moreover, Hermes is able to recognize sounds, localize a resource and tracking supports. The evaluation of the sample was based on scenarios, where the starting point was a resource chosen by the user, showing that the application supports accessibility. Hermes application was evaluated by 10 users, one of them is deaf. The evaluators approved the requirement of ease to use in $88 \%$, and the requirement of usefulness in $90 \%$.
\end{abstract}

Keywords: Accessibility Ubiquitous, Hearing impaired, Ubiquitous Computing.

\section{Introdução}

Nos últimos anos, a variedade de dispositivos móveis e o crescente uso das redes de comunicação sem fio vêm estimulando cada vez mais a adoção da computação móvel e de serviços que explorem suas características. Entretanto, nota-se que tais serviços não estão aptos a atender as pessoas com determinadas deficiências, dentre as quais estão os deficientes auditivos. O Instituto Brasileiro de Geografia e Estatística - IBGE [1], no censo realizado no ano de 2000, estimou que 5,7 milhões de brasileiros possuem algum tipo de deficiência auditiva e, no censo de 2010 [2], esse número subiu para 9,7 milhões e entre essas estimativas mais de 2 milhões são consideradas totalmente surdas.

\footnotetext{
${ }^{1}$ Programa de Pós-Graduação em Computação Aplicada, - Unisinos - São Leopoldo (RS) - Brasil

\{felipedifrr.edu.br\}

${ }^{2}$ Programa de Pós-Graduação em Computação Aplicada, - Unisinos - São Leopoldo (RS) - Brasil

$\{j$ barbosa@unisinos.br\}
}

http://dx.doi.org/10.5335/rbca.v8i3.5816 
Por meio desses dados, pode-se perceber a importância de inclusão social para pessoas com defíciência auditiva. Para Tavares [3], cidadãos com algum tipo de deficiência enfrentam dificuldades no dia a dia devido à falta de recursos para acessibilidade. Eles precisam de suporte para que possam efetivamente exercer a cidadania plena.

A computação ubíqua busca tornar as tarefas que fazem uso de computação transparentes aos usuários, considerando seu ambiente natural [4]. De acordo com Satyanarayanan [5], a Computação Ubíqua pode ser considerada uma extensão da computação móvel, pois herdou algumas de suas características com a adição de aspectos próprios, como sensibilidade ao contexto e invisibilidade. Quando se lida com contextos podem ser identificadas entidades, tais como: lugares (salas, prédios), pessoas (indivíduos, grupos) e coisas (objetos físicos e recursos computacionais) [6]. Cada uma dessas pode ser descrita por meio de vários atributos, como: identidade (tendo um único identificador), localização (uma entidade possui uma localização e proximidades), status (corresponde às propriedades intrínsecas de cada entidade) e tempo (usado para definir a situação e ordenação de eventos).

Segundo Wagner [7], a adaptação em contextos deve compreender a situação do usuário e seu comportamento de acordo com as informações disponibilizadas. Contudo, não é apenas útil saber o contexto em que o usuário se encontra, mas saber a história de contextos visitados. Essa informação pode ser valiosa para ajudar a aplicação a se adaptar ao utilizador. Essa história de contextos é chamada de Trilhas. Porém, Wagner [7] afirma que para uma aplicação adaptar-se de forma eficaz a um usuário, é importante não só conhecer a história de contextos visitados pelo usuário, mas, também, saber quem é o usuário, ou seja, quais são suas características. Esse tipo de sistema organiza as características dos usuários em perfis de usuários.

Este artigo propõe um modelo de acessibilidade ubíqua para suporte ao deficiente auditivo, denominado Hermes $^{3}$. O Hermes é uma extensão do modelo genérico chamado Hefestos ${ }^{4}$ [3], o qual enfoca diversos padrões de acessibilidade sem restrição quanto ao tipo de deficiência. O Hermes é baseado no modelo Tirésias [8] que também é uma extensão do Hefestos, no entanto, é, especificamente, orientado ao deficiente visual.

O artigo está organizado em sete seções. A Seção 2 apresenta o modelo Hermes, destacando sua interação com o Hefestos. A Seção 3 descreve o protótipo. A Seção 4 aborda a avaliação de funcionalidade do modelo por meio de cenários. A Seção 5 avalia a usabilidade do protótipo. A seção 6 discute os trabalhos relacionados. Por fim, a Seção 7 apresenta as considerações finais.

\section{Modelo Hermes}

A Figura 1 mostra a arquitetura do modelo Hermes, a qual é composta por quatro módulos (Recursos, Eventos, Monitor de Som, Configuração) e um Agente Assistente Pessoal (AAP). O AAP é uma especialização do assistente pessoal proposto pelo Hefestos [3], focado na acessibilidade de pessoas com deficiência auditiva. Por meio desse agente, o Hermes acessa o Hefestos para obtenção de informações como os perfis de usuários, recursos disponíveis nos contexto [9] e históricos de locais visitados pelo usuário.

O módulo de recursos sugere alguns que possam auxiliar o deficiente auditivo criando rotas com base na localização do usuário. O módulo de eventos informa ao usuário sobre acontecimentos ao seu redor utilizando componentes do dispositivo como o vibracall e luzes do flash do smartphone. O módulo monitor de som possui duas funcionalidades. A primeira permite o reconhecimento de som em ambientes por meio do cálculo do Nível de Pressão Sonora que o usuário poderá ser alertado de acordo com o patamar estabelecido no Módulo de Configurações em Decibéis $(\mathrm{dB})$. A segunda funcionalidade consiste no reconhecimento de voz para reconhecimento da fala de um locutor próximo ao usuário. O módulo de configuração é responsável pela adaptação do Hermes ao usuário para melhor customização. Por fim, o Agente Assistente Pessoal é responsável por interagir com o Hefestos na busca de informações como o perfil do usuário, recursos disponíveis, busca de perfis disponíveis por localização e o gerenciamento de histórico. As próximas seções descrevem cada módulo do Hermes.

\footnotetext{
${ }^{3} \mathrm{Na}$ mitologia grega, Hermes era o deus mensageiro.

${ }^{4}$ Deus da mitologia grega portador de deficiência física, o qual construía máquinas para superação de sua deficiência.
} 
Figura 1: Arquitetura do modelo Hermes

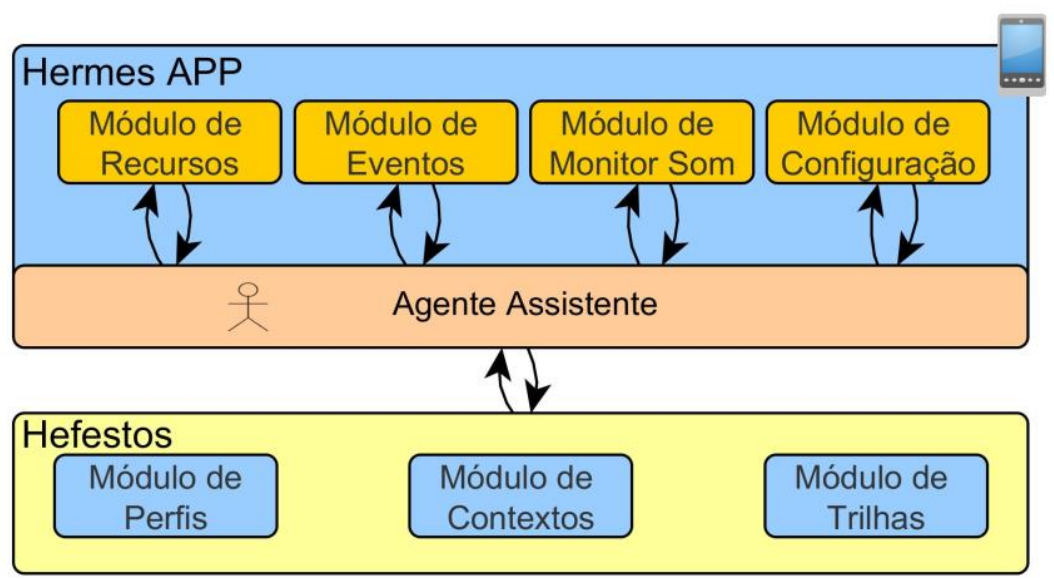

Fonte: elaboração dos autores com base nos dados da pesquisa.

\subsection{Módulo de recursos}

O módulo de Recursos disponibiliza informações oriundas do Hefestos ao deficiente auditivo. Essas informações são indicações de recursos de acessibilidade próximos à localização do usuário. $\mathrm{O}$ módulo de recursos também cria um caminho ou rota para acessar um determinado recurso escolhido pelo usuário. Essa interação é feita por meio de mapas, facilitando a compreensão do usuário para chegar ao seu destino. Atualmente, ainda existe uma carência significativa no que diz respeito a atendimento de qualidade para o deficiente auditivo. Ou seja, sem a utilização de Libras, o deficiente é prejudicado, pois sem a comunicação por meio da língua mãe, ele não compreende claramente o que lhe é dito, limitando sua comunicação e o desejo de retorno a um ambiente.

Ao entrar em um contexto, o Hermes informa ao deficiente auditivo se o ambiente tem recursos de acessibilidade como um intérprete de Libras no local ou serviços de apoio específicos como tradução na comunicação. Assim, o deficiente se sente mais seguro e satisfeito pela obtenção de algum tipo de ajuda de alguém que lhe compreenda.

\subsection{Módulo de eventos}

O módulo de eventos informa algum acontecimento oriundo do módulo de monitor de Som. Para que essas informações chamem a atenção do usuário, o módulo de eventos suporta a funcionalidade de alertas vibratórios. A vibração é usada para notificar ao usuário o fato de que houve a deteç̧ão de um som ou um reconhecimento de voz e essas informações são mostradas no visor.

Outra funcionalidade que o módulo de eventos suporta é o alerta luminoso. Quando o usuário recebe uma informação e não está com seu smartphone em mãos ou no bolso, mas em uma mesa que esteja próxima, a luzes do flash da câmera ficam piscando. O alerta luminoso somente será interrompido quando o usuário acessar a informação.

\subsection{Módulo monitor de som}

O módulo monitor de som apresenta duas funcionalidades para oferecer suporte ao deficiente auditivo: o reconhecimento de som e o reconhecimento de voz.

\subsubsection{Reconhecimento de som}

O reconhecimento de som é baseado no nível de pressão sonora cuja medida serve para determinar o grau de potência de uma onda sonora, tendo como unidade logarítmica o decibel $(\mathrm{dB})$. O reconhecimento de som atua 
como um decibelímetro baseado no algoritmo para cálculo de nível de pressão sonora para ajudar a verificar a intensidade do som. Conforme Braga [11], após a medição sobre a intensidade de sons comuns é possível fazer uma escala entre o limiar de audibilidade $(0 \mathrm{~dB})$ e o limiar de dor $(>140 \mathrm{~dB})$. Baseando-se nessa escala, o deficiente auditivo pode ser notificado de eventos ao seu redor de acordo com o patamar configurado no módulo de configuração.

\subsubsection{Reconhecimento de voz}

O reconhecimento de voz permite a detecção da voz de um locutor, tendo como objetivo a determinação automática do indivíduo emissor de uma determinada locução, materializada em sinal de voz [12]. Quando o usuário habilita o reconhecimento de voz, a funcionalidade, que usa o microfone do smartphone, permite reconhecer pequenas frases e palavras de um locutor próximo ao usuário sem a necessidade de um padrão de voz. Durante o processo de reconhecimento, a funcionalidade reconhece a fala e repassa para o módulo de eventos essa palavra ou pequena frase para que o usuário saiba o que está sendo informado.

\subsection{Módulo de configuração}

O módulo de configuração permite a customização das funcionalidades do Hermes. O usuário pode: (1) configurar o tempo de vibrações em um evento recebido; (2) configurar o intervalo de vibrações; (3) habilitar sinais luminosos; (4) configurar o nível de alerta em decibéis para o reconhecimento de som; (5) ativar ou desativar as funcionalidades do módulo de monitor de som; (6) o tipo de linguagem em que o usuário gostaria de receber pelo módulo de eventos, nesse caso a língua portuguesa.

\subsection{Agente assistente pessoal}

O agente assistente pessoal é baseado no Tirésias [8] e foi modelado usando a metodologia Prometheus [13], como mostra a Figura 2. A estrela representa a percepção suportada pelo agente assistente pessoal. As flechas são ações realizadas pelo agente de acordo com a percepção do usuário. Os envelopes representam as mensagens trocadas entre os agentes, os quais são representados por um retângulo.

Para gerar a comunicação com o Hefestos, o agente assistente pessoal propõe a troca de informações por meio de webservices. O Agente Assistente é responsável por receber a localização atual do usuário, retornando recursos disponíveis para o usuário.

Figura 2: Modelagem do agente assistente pessoal

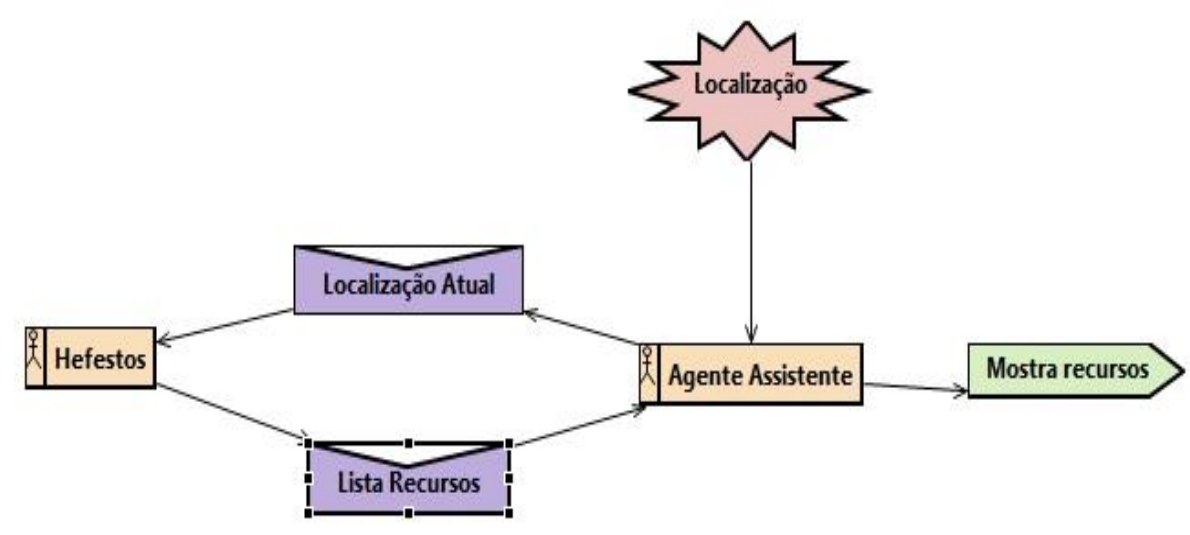

Fonte: elaboração dos autores com base nos dados da pesquisa.

O App Hermes, além de ter sensibilidade aos contextos, permite o registro de históricos de uma entidade. No caso do App Hermes, as trilhas representam os históricos de contextos e, principalmente, as rotas do usuário [14]. 


\section{Protótipo Hermes}

Um protótipo do modelo Hermes foi desenvolvido para dispositivos móveis que usam o sistema operacional Android. Para o reconhecimento de voz, foi utilizado a API da Google, o Speech Recognition e para o reconhecimento de som, foi incluído um algoritmo para medir o nível de pressão sonora desenvolvido por Ramirez [15]. Para a avaliação do protótipo, foi usado um cenário real na cidade de Boa Vista - Roraima. Esse cenário disponibilizou recursos para suporte ao deficiente auditivo. A Figura 3 mostra o mapa da área de abrangência do Hermes App e a Tabela 2 apresenta a descrição da lista de recursos mapeados. No Hefestos, seria incluso 5 uma nova deficiência chamada de "Deficiência Auditiva", recursos de suporte ao deficiente e novos usuários com perfil de deficiência auditiva.

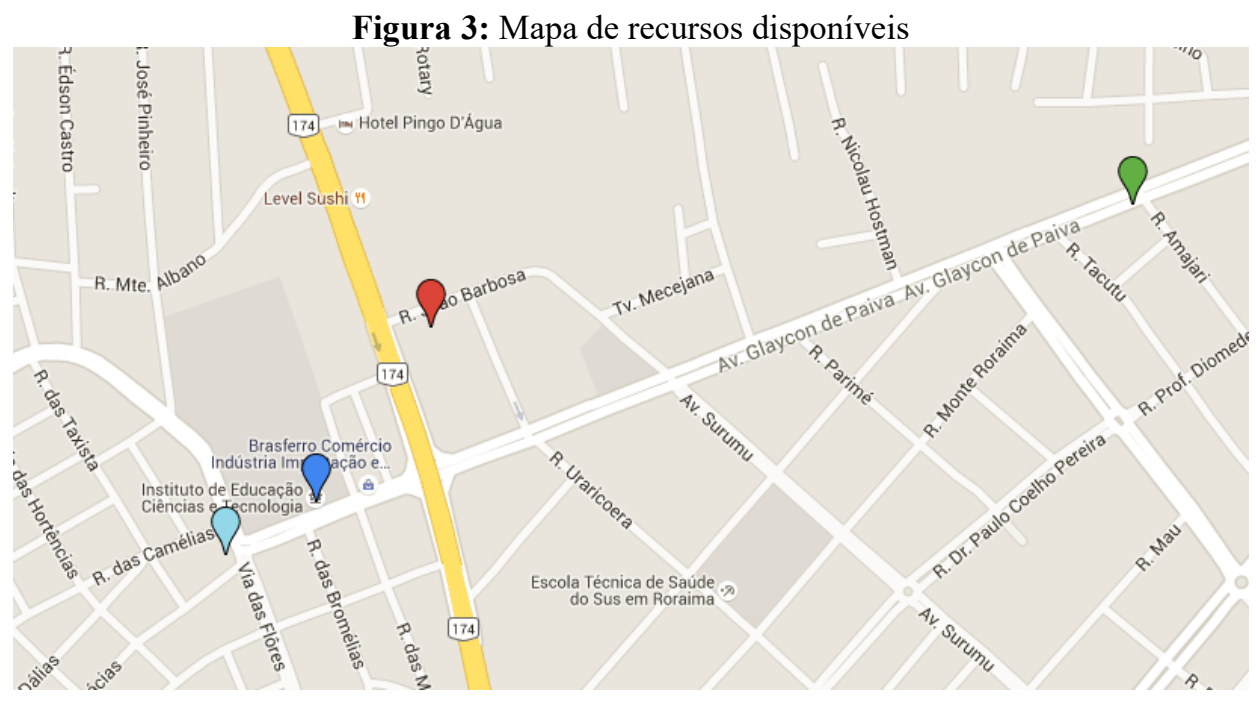

Fonte: elaboração dos autores com base nos dados da pesquisa.

Tabela 1: Descrição dos recursos e localização

\begin{tabular}{l|l|c|c}
\hline \multicolumn{1}{c|}{ Nome do Recurso } & \multicolumn{1}{|c|}{ Descrição do Recurso } & Latitude & Longitude \\
\hline IFRR & Coordenação de Núcleo de Inclusão & 2.81299 & -60.69389 \\
\hline Escola Dom José Nepote & Secretaria Escolar & 2.81225 & -60.69519 \\
\hline Sesc Mecejana & Núcleo de Inclusão Social & 2.81547 & -60.69226 \\
\hline Hospital Unimed & Assistência Social & 2.81719 & -60.68228 \\
\hline
\end{tabular}

Fonte: elaboração dos autores com base nos dados da pesquisa.

A Figura 4a apresenta a tela inicial do Hermes App na qual são inseridos um usuário e uma senha. A tela principal (Figura 4b), por padrão, inicia no módulo de monitor de som (reconhecimento de som) e avisa por meio do módulo de eventos sobre qualquer acontecimento ao redor do deficiente auditivo. Ainda no módulo de monitor de som, o reconhecimento de voz (Figura 4c) poderá ser ativado por intermédio do botão de microfone.

\footnotetext{
${ }^{5}$ As funcionalidades do Hefestos foram simuladas. Sendo assim, os testes avaliaram a funcionalidade do Hermes APP.
} 

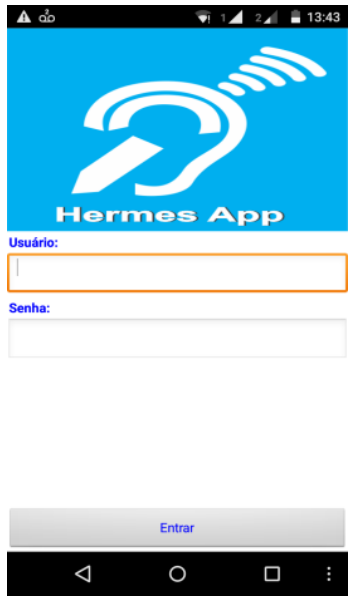

(a) Tela inicial
Figura 4: Telas do Hermes App
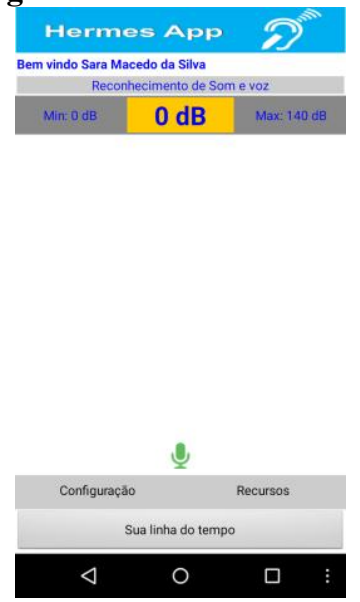

(b) Tela principal
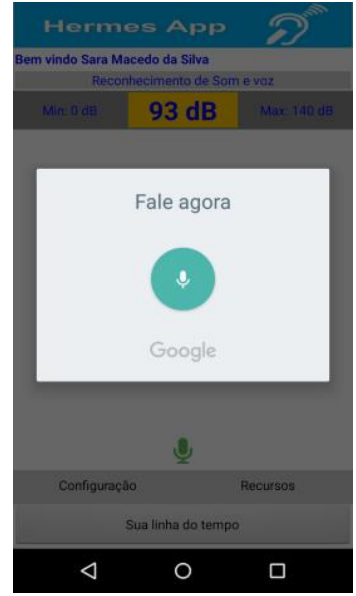

(c) Reconhecimento de voz

Fonte: elaboração dos autores com base nos dados da pesquisa.

A Figura 5a mostra a tela do Módulo de Recursos, que disponibiliza uma lista de recursos próximos à localização atual do usuário (Figura 5b). Em seguida, o usuário escolhe um recurso e o Hermes App cria uma rota até o destino.

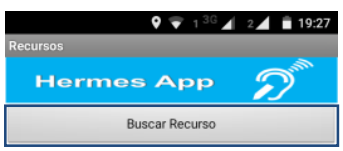

Figura 5: Módulo de recursos

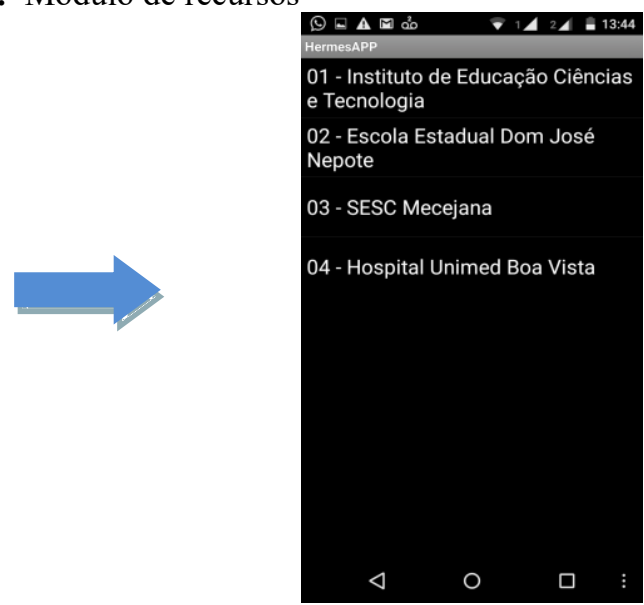

(a) Tela de recursos

(b) Lista de recursos

Fonte: elaboração dos autores com base nos dados da pesquisa.

O Módulo de Configuração permite customizar uma série de parâmetros do Hermes App como o nível de alerta (Figura 6a e Figura 6b), que serve para alertar o usuário no reconhecimento de som do módulo de monitor de Som. Há outras configurações como: duração da vibração, pausa da vibração, sinais luminosos, ativar ou desativar o reconhecimento de voz e de som. O Módulo de configuração ainda permite ajustar o tipo de linguagem que o usuário gostaria de receber, nesse caso, em língua portuguesa. 


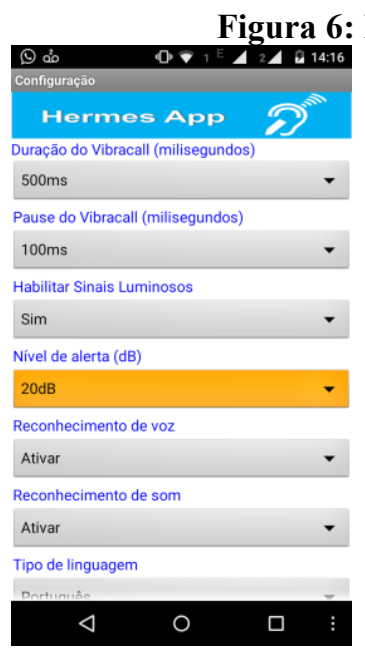

(a) Tela de configuração

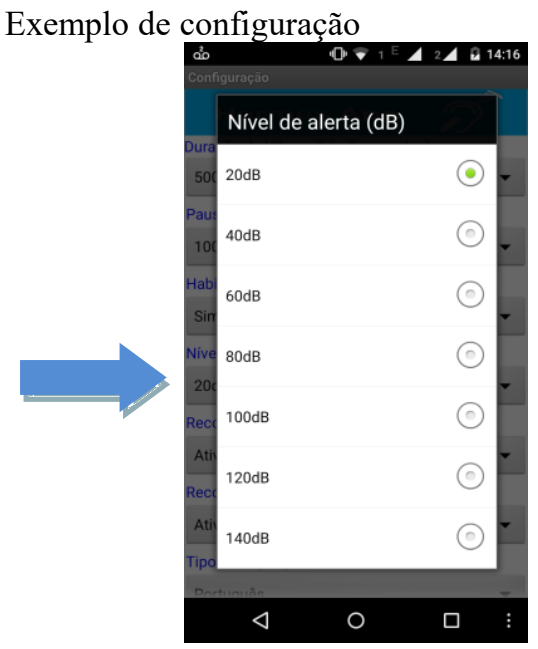

(b) Definindo nível de alerta

Fonte: elaboração dos autores com base nos dados da pesquisa.

\section{Avaliação de funcionalidade por meio de cenários}

A comunidade científica tem empregado cenários para avaliação de aplicações ubíquas e sistemas sensíveis de contexto [5]. Partindo dessa estratégia, foram criados dois cenários para avaliação do Hermes os quais foram executados por um usuário ${ }^{6}$, portando um smartphone Moto $\mathrm{G} 2^{\circ}$ Geração. $\mathrm{O}$ primeiro cenário mostra o usuário caminhando pela cidade de Boa Vista, RR, e sua interação inicial com o Hermes é a localização de recursos próximos. No segundo cenário, o usuário explora funcionalidades do Hermes por meio do módulo de monitor de som para reconhecimento de som e de voz.

\subsection{Cenário 1}

"Ana de 22 anos é portadora de deficiência auditiva. Ela ficou surda com um ano de idade e até hoje não sabe o que a levou a surdez. Atualmente, Ana possui o conhecimento na Língua Brasileira de Sinais - Libras e também conhece um pouco da língua portuguesa. Ela usa o Hermes App no seu smartphone. Ana está caminhando pela cidade de Boa Vista em Roraima e logo fica perdida em um bairro, pois ela esta tentando chegar a uma Vila Olímpica. Ela tenta pedir informações de pessoas que ali trafegavam, mas por ser deficiente auditiva, as pessoas não entendiam a linguagem de sinais que Ana fazia para obter uma informação. Então Ana utilizou o Hermes para buscar recursos de acessibilidade disponíveis próximos a sua localização. Em seguida, o Hermes fornece uma lista (Figura 7a) ordenada pela proximidade da localização de Ana. Então ela decide buscar pelo primeiro recurso. O Instituto Federal de Roraima (IFRR) foi o recurso escolhido por Ana. Em seguida, o Hermes traçou uma rota até o destino escolhido (Figura 7b). Ao chegar ao IFRR, Ana recebeu um alerta vibratório pelo Hermes mostrando detalhes do recurso escolhido (Figura 7c). Ana, então, recebe a confirmação de que está no recurso desejado e localiza a Coordenação de Núcleo de Inclusão do Instituto Federal de Roraima. Ana foi atendida pelo professor interprete que tinha conhecimento na língua de sinais. Ana conheceu recursos de acessibilidade próximos a sua localização e pegou a informação que ela precisava para chegar à vila olímpica."

\footnotetext{
${ }^{6} \mathrm{O}$ usuário era portador de deficiência auditiva.
} 


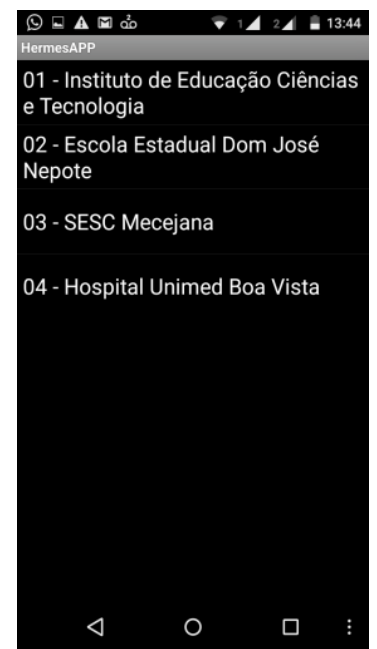

(a) Lista de recurso
Figura 7: Detalhe do recurso

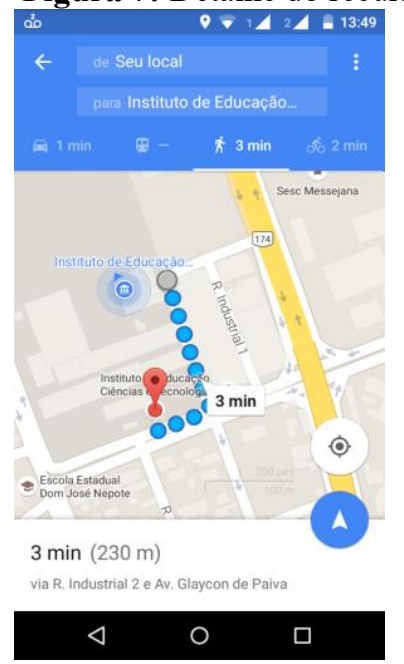

(b) Rota até o recurso

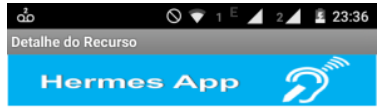

Coordenação de Núcleo de Inclusão IFRR

Latitude: 281299

Longitude: -60.69389

Fonte: elaboração dos autores com base nos dados da pesquisa.

A Tabela 2 resume a dinâmica do cenário, destacando os atores e as ações.

Tabela 2: Dinâmica do primeiro cenário

\begin{tabular}{l|l}
\hline \multicolumn{1}{c|}{ Ator } & \multicolumn{1}{c}{ Ação } \\
\hline Usuário & Busca recurso \\
\hline Hermes App & $\begin{array}{l}\text { Considerando a localização do usuário, o Hermes se comunica com o servidor para simular a } \\
\text { interação com o Hefestos e recebe a lista de recursos próximos, sendo que a lista é } \\
\text { disponibilizada em ordem de proximidade em relação ao ponto em que o usuário está. }\end{array}$ \\
\hline Usuário & Verifica a lista e escolhe o recurso de acordo com seu interesse. \\
\hline Hermes App & O Hermes mostra o mapa com a indicação do recurso e habilita a opção de rota. \\
\hline Usuário & O usuário seleciona a opção de rota até o destino. \\
\hline Hermes App & $\begin{array}{l}\text { O Hermes cria a rota com base na localização atual do usuário até o destino escolhido por } \\
\text { esse. }\end{array}$ \\
\hline Usuário & O usuário chega ao destino. \\
\hline Hermes App & Começa a emitir um alerta vibratório com a informação detalhada do recurso. \\
\hline Hermes App & $\begin{array}{l}\text { Ao detectar que o usuário chegou ao recurso, o Hermes comunica com o Hefestos para } \\
\text { salvar o local visitado. }\end{array}$ \\
\hline
\end{tabular}

Fonte: elaboração dos autores com base nos dados da pesquisa.

Conforme descrito na Tabela 2, nota-se que o aplicativo Hermes APP tem uma dinâmica de interação e comunicação com o deficiente auditivo, pois esse alerta ao usuário que está no local selecionado inicialmente. Outro ponto a ser destacado dentro dessa tabela é que o aplicativo cria, automaticamente, um registro do local visitado, criando um histórico de navegação (Figura 8), o qual pode ser visualizado pelo usuário por meio do aplicativo, servindo como orientação para ir, novamente, àquele lugar registrado e visitado por ele, caso ele não conheça suficientemente a cidade. 
Figura 8: Tela de histórico
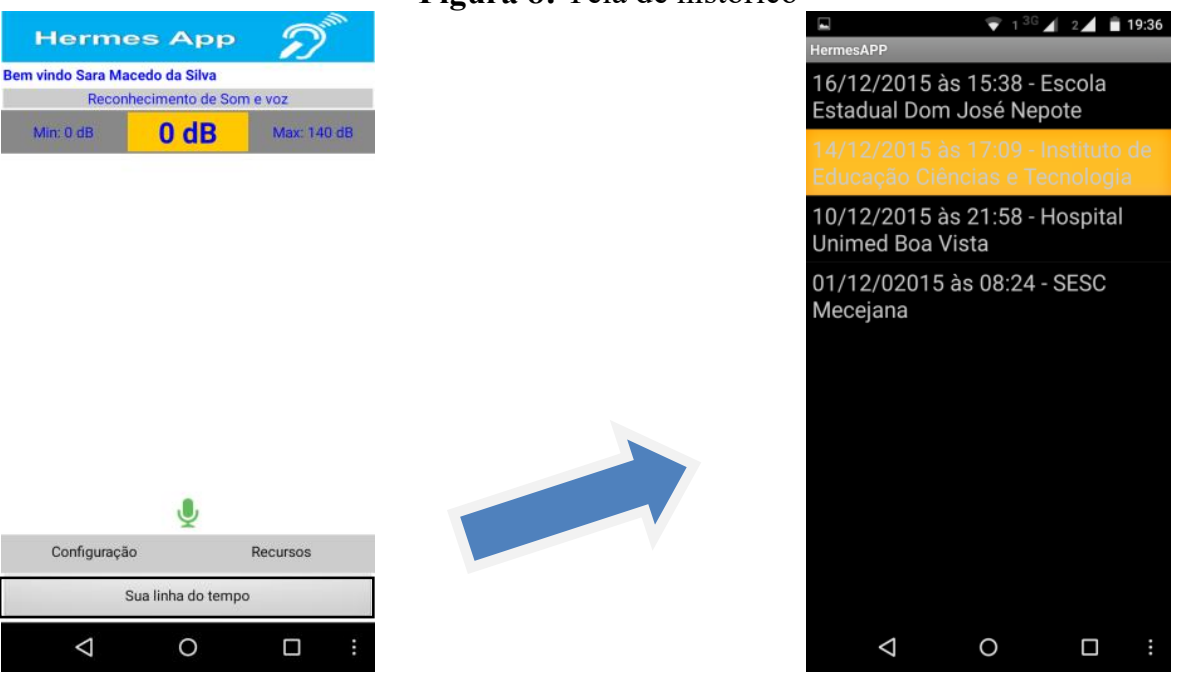

Fonte: elaboração dos autores com base nos dados da pesquisa.

\subsection{Cenário 2}

"Ana quando chega à escola do IFRR, customiza no Módulo de Configuração o nível de alerta em Decibéis (Figura 9a) no qual ela deseja ser avisada sobre algum ruído que aconteça ao seu redor. Ana colocou o nível de alerta a partir de $90 \mathrm{~dB}$, que é considerado como um som alto. Ela caminha em direção à Coordenação de Núcleo de Inclusão e, nesse momento, ela sente a vibração do seu smartphone alertando com um ruído que algo foi detectado (Figura 9b). Ana fica atenta e começa a procurar aquele som que atingiu o patamar configurado no Hermes. Quando Ana ficou atenta àquele som, imediatamente ativou o reconhecimento de voz (Figura 10a). Um aluno da escola passa bem próximo à Ana e emite uma fala de 'cuidado saia daí' (Figura 10b). Ana sente novamente as vibrações do smartphone e verifica que alguma pessoa falou uma pequena frase próxima a ela. Imediatamente Ana se afasta do lugar em que ela estava e logo em seguida percebe que pessoas estavam fazendo serviços de reforma no prédio do IFRR. Nesse momento Ana soube o que estava acontecendo ao seu redor e o Hermes foi capaz de oferecer esse suporte referente a qualquer acontecimento à Ana."

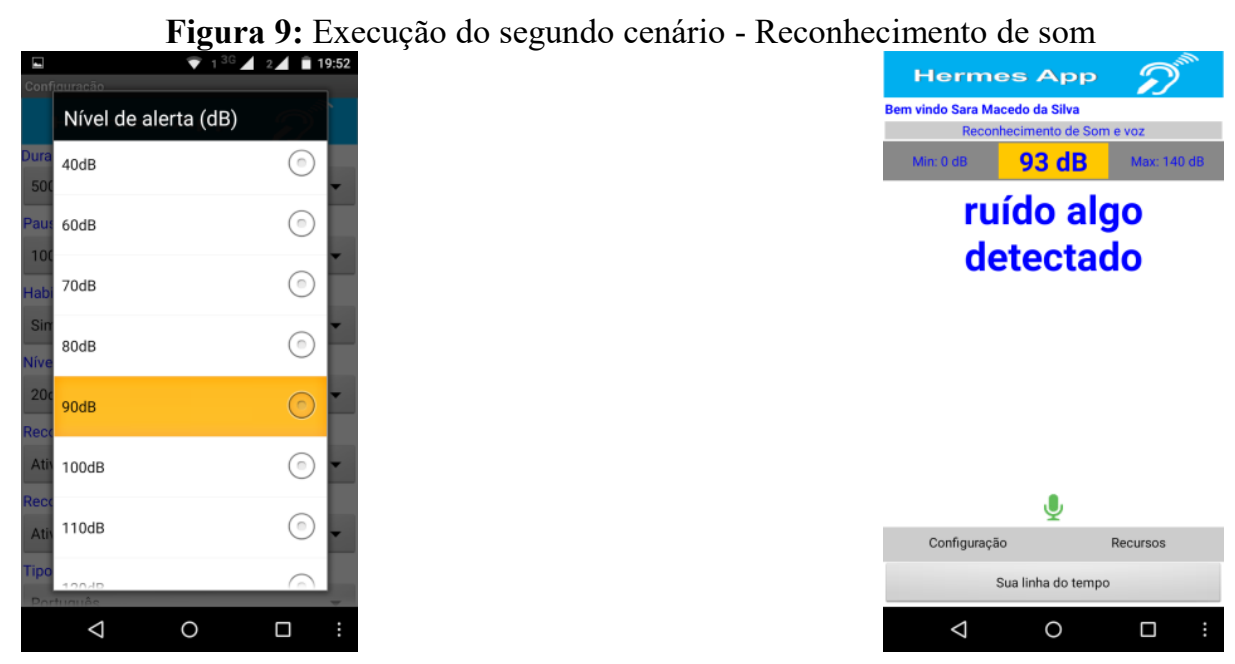

(a) Configuração de nível de alerta

(b) Reconhecimento de som

Fonte: elaboração dos autores com base nos dados da pesquisa. 
Figura 10: Execução do segundo cenário - Reconhecimento de voz

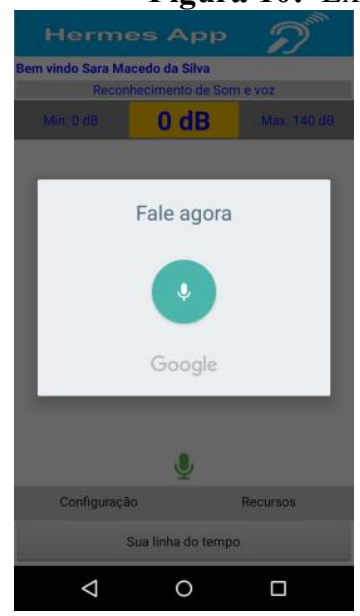

(a) Ativando reconhecimento de voz
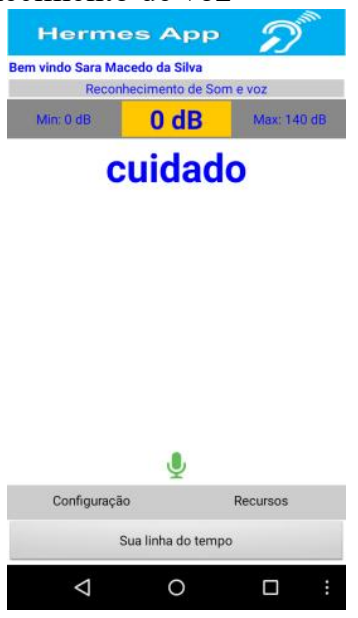

(b) Alerta de reconhecimento de voz

Fonte: elaboração dos autores com base nos dados da pesquisa.

A Tabela 3 resume a dinâmica do cenário, destacando os atores e as ações.

Tabela 3: Dinâmica do segundo cenário

\begin{tabular}{l|l}
\hline \multicolumn{1}{c|}{ Ator } & \multicolumn{1}{c}{ Ação } \\
\hline Usuário & Configura um patamar em decibéis para ser alertado. \\
\hline Hermes App & $\begin{array}{l}\text { O monitor de som fica fazendo o cálculo de pressão sonora a todo tempo. Até que esse } \\
\text { chegue ao valor configurado pelo usuário, para enviar o alerta por meio do módulo de } \\
\text { eventos. }\end{array}$ \\
\hline Usuário & $\begin{array}{l}\text { Recebe a informação por intermédio de alertas vibratórios. Em seguida, o usuário ativa o } \\
\text { reconhecimento de voz. }\end{array}$ \\
\hline Hermes App & $\begin{array}{l}\text { Hermes desabilita o reconhecimento de som para reconhecer a voz. Em seguida, reconhece a } \\
\text { voz e manda a informação por meio do módulo de eventos. }\end{array}$ \\
\hline Usuário & $\begin{array}{l}\text { Recebe a informação por meio de alertas vibratórios. O usuário fica atento a qualquer } \\
\text { acontecimento ao seu redor. }\end{array}$ \\
\hline
\end{tabular}

Fonte: elaboração dos autores com base nos dados da pesquisa.

De acordo com a Tabela 3, pode-se verificar que o aplicativo disponibiliza configurações para reconhecimento de ambiente, nesse caso, ruídos. O aplicativo realiza uma comunicação com o deficiente auditivo, alertando sobre perigos, graças aos itens de configuração do reconhecimento de voz em sua volta e ao som que é transmitido por vibrações e, ainda, à mensagem de texto.

\section{Avaliação de usabilidade}

Para fazer a avaliação de aceitação do modelo Hermes, foram elaborados questionários com base nos conceitos do modelo de aceitação de tecnologia proposto por Davis [16] chamado de Technology Acceptance Model - TAM. Esse modelo considera a facilidade percebida de uso, que trata a respeito da percepção do usuário em relação a quanto uma tecnologia pode minimizar esforços humanos. O TAM também considera a utilidade percebida que trata a respeito da percepção do usuário sobre o quanto a tecnologia pode melhorar o seu desempenho. Ambas as percepções influenciam na aceitação de uma nova tecnologia. 
As avalições contaram com a participação voluntária de dez sujeitos, sendo que somente um é portador de deficiência auditiva - uma aluna - e os demais participantes eram servidores do IFRR. Os nove voluntários não deficientes usaram um dispositivo no ouvido para simular a perda de audição.

O procedimento adotado para o recrutamento de participantes ocorreu por meio de convite de acordo com a conveniência do experimento, não havendo embasamento estatístico para a proporção considerada entre o número de usuários com deficiência e o número de usuários sem deficiência.

Os participantes utilizaram o Hermes APP, testaram as funcionalidades do protótipo e, em seguida, responderam o questionário proposto. No questionário, foi utilizado a escala de Likert, na qual os participantes especificam seu nível de concordância plena ou discordância total. Nesse questionário, foram empregados os seguintes níveis de afirmação na escala: discordo plenamente, discordo parcialmente, nem concordo nem discordo, concordo parcialmente e concordo plenamente. Os questionários são apresentados nas Tabelas 4 e 5.

Tabela 4: Itens do questionário sobre facilidade de uso

\begin{tabular}{c|l}
\hline Item & Facilidade percebida de uso \\
\hline 1 & As informações de recursos são apresentadas de forma clara, sendo de fácil compreensão. \\
\hline 2 & É fácil a compreensão de como buscar recursos de acessibilidade. \\
\hline 3 & As informações sobre eventos são apresentadas de forma clara, quando reconhecido algum som. \\
\hline 4 & Não é necessário muito esforço para configurar o Hermes. \\
\hline 5 & O Hermes App facilita a identificação de sons. \\
\hline
\end{tabular}

Fonte: elaboração dos autores com base nos dados da pesquisa.

Tabela 5: Itens do questionário sobre utilidade percebida

\begin{tabular}{c|l}
\hline Item & Utilidade percebida \\
\hline 1 & Os recursos para acessibilidade foram uteis para a minha necessidade. \\
\hline 2 & O Hermes facilita a identificação de recursos para acessibilidade em ambientes. \\
\hline 3 & O Hermes seria útil para oferecer suporte aos portadores de deficiência auditiva. \\
\hline 4 & A utilidade de deteção de voz é útil para apoiar o deficiente auditivo. \\
\hline 5 & $\begin{array}{l}\text { O histórico de locais visitados me ajudou a recuperar um recurso que não estava disponível por causa de } \\
\text { minha localização atual. }\end{array}$ \\
\hline
\end{tabular}

Fonte: elaboração dos autores com base nos dados da pesquisa.

A estrutura das respostas foi organizada em gráficos, com percentuais para melhor compreensão de nota de aprovação.

Ao analisar os itens que compõem a parte do questionário referente à facilidade percebida de uso, observa-se, conforme está representado na Figura 11 que, $88 \%$ aprovaram e consideraram o Hermes APP um aplicativo com facilidade percebida de uso, ou seja, sem dificuldades encontradas para o manuseio. Desse percentual alcançado, $50 \%$ disseram que concordavam totalmente e $38 \%$ concordavam parcialmente. Apenas $2 \%$ discorda parcialmente já que é necessário muito esforço para configurar o Hermes e $10 \%$ respondeu que não concorda nem discorda. Não houve resposta quanto ao discordo totalmente.

Em relação à utilidade percebida, foram tabulados os seguintes percentuais: $58 \%$ de concordo totalmente e $32 \%$ concordo parcialmente, totalizando, assim, $90 \%$ de aprovação quanto à sua utilidade. Apenas $10 \%$ disseram que não concordam e nem discordam. Logo, entende-se que o Hermes APP teve uma boa avaliação no requisito de aprovação quanto à utilidade percebida. Confira na Figura 12. 
Figura - 11 Facilidade percebida de uso

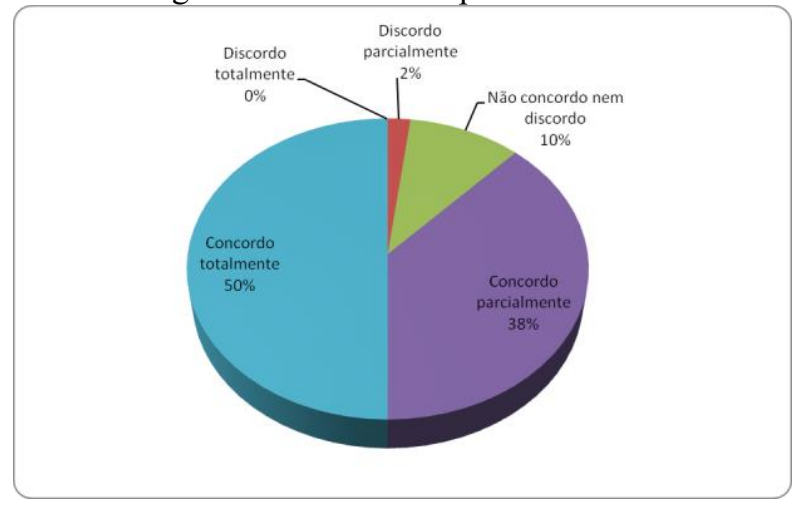

Figura - 12 Utilidade percebida

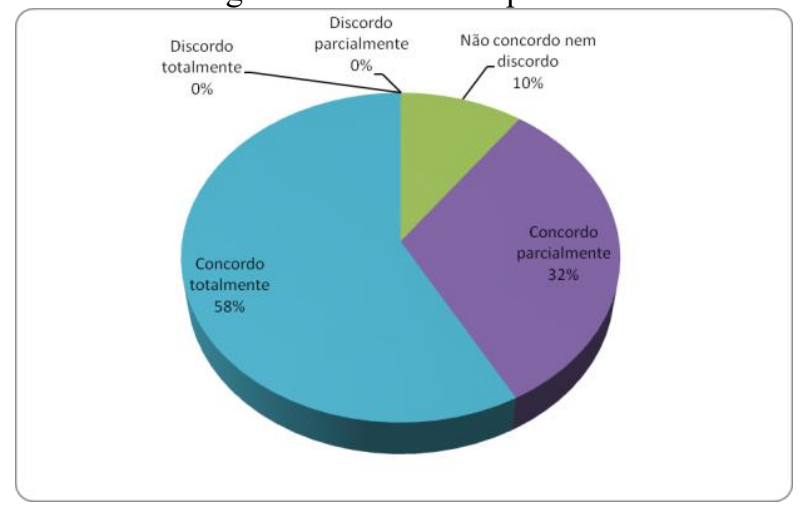

Fonte: elaboração dos autores com base nos dados da pesquisa.

\section{Trabalhos relacionados}

ProDeaf [17] é um aplicativo para smartphone que suporta dicionário e tradução para Libras. Além das funções de tradução de palavras e de pequenas frases, a plataforma permite que sejam criados conteúdos em Libras, bem como sejam realizados acréscimos de vocabulários como novos sinais. No ProDeaf os sinais são executados por um personagem animado, um avatar. O ProDeaf propõe a comunicação entre deficientes auditivos e ouvintes não usuários da língua de sinais, promovendo a interação por meio da tradução entre a língua de sinais e a língua falada.

O sistema de Tecnologia Assistiva para Auxílio a Deficientes Auditivos e Surdos (TAADA)[18] usa tecnologia de reconhecimento de voz e palavras selecionadas por meio do dispositivo móvel, atuando o smartphone como interface entre o deficiente auditivo e o ouvinte. A TAADA permite chamar a atenção do deficiente por meio de alertas sonoros e recursos de vibracall ou de sinais luminosos no display do aparelho, sempre que alguém chame o deficiente auditivo pelo nome previamente registrado no dicionário de dados do sistema.

Hand Talk [19] é um aplicativo que converte conteúdos em texto e voz para a linguagem de sinais para surdos, em tempo real. A solução também converte imagens para Libras. Dentro desse contexto, o Hand Talk usa o auxílio de um interprete virtual, o Hugo, um personagem em 3D que torna a utilização da solução interativa e de fácil compreensão.

Rybená [20] é um aplicativo desenvolvido pelo Grupo ICTS, usado como tradutor de Libras e sintetizador de voz capaz de traduzir textos do português para Libras e de converter português escrito para voz falada no Brasil. O Rybená também tem um personagem 3D para fazer a interpretação do português para Libras. As últimas trinta frases são armazenadas no histórico, no intuito de ser reutilizadas de uma maneira mais rápida.

O projeto Mielke [21] é um sistema para usar algoritmos de reconhecimento de som ambiental para detectar eventos de áudio relevantes no tráfego rodoviário. A contribuição deste trabalho consiste na aplicação de técnicas de reconhecimento de padrões de tecnologia de comunicação para implementar um sistema que analisa o ambiente e os sinais de eventos acústicos classificados como críticos para o usuário surdo. $\mathrm{O}$ algoritmo verifica os sinais de frequência e a lógica de uma sirene e notifica o usuário.

Foram selecionados critérios, descritos a seguir, para uma comparação entre os trabalhos relacionados. (1) Sensível ao contexto: indica se o sistema possui dados de contexto conforme proposto por Dey [9]; (2) Configuração: indica se o sistema apresenta variedade de customização de funcionalidades, tais como, configuração do tipo de linguagem a ser alertada pelo evento, tempos de vibração, alertas luminosos do display e do flash do smartphone; (3) Reconhecimento de som em ambientes: indica se o sistema tem reconhecimento de som ou ruídos em decibéis; (4) Localização de recursos: indica se o sistema considera a localização de recursos próximos ao usuário e a localização de perfis de ajuda; (5) Suporte a trilhas: indica se foi empregada alguma forma de gerenciamento de históricos de contextos conforme [14]; (6) Perfil: avalia se foi utilizado algum tipo de gerenciamento de perfis de usuários. A Tabela 6 apresenta um comparativo entre eles. 
Tabela 6: Comparação entre os trabalhos relacionados

\begin{tabular}{l|c|c|c|c|c|c}
\hline \multicolumn{1}{c|}{ Trabalhos } & $\begin{array}{c}\text { ProDeaf } \\
{[17]}\end{array}$ & $\begin{array}{c}\text { TAADA } \\
{[18]}\end{array}$ & $\begin{array}{c}\text { Hand } \\
\text { Talk[19] }\end{array}$ & Rybená[20] & $\begin{array}{c}\text { Tecnologia } \\
\text { assistiva } \\
\text { para } \\
\text { pessoas com } \\
\text { deficiência } \\
\text { auditiva[21] }\end{array}$ & Hermes \\
\hline Sensível ao contexto & Não & Não & Não & Não & Não & Sim \\
\hline Configuração & Não & Sim & Não & Não & Não & Sim \\
\hline Reconhecimento de som & Não & Não & Não & Não & Não & Sim \\
\hline Localização de Recurso & Não & Não & Não & Não & Não & Sim \\
\hline Suporte a Trilhas & Não & Não & Não & Não & Não & Sim \\
\hline Perfil & Não & Sim & Não & Não & Sim & Sim \\
\hline
\end{tabular}

Fonte: elaboração dos autores com base nos dados da pesquisa.

Nenhum dos trabalhos tem uma lista de recursos disponíveis baseado na localização do usuário. Com relação ao suporte de trilhas, ou seja, o histórico de locais visitados, nenhum dos trabalhos relacionados possui esse suporte. No quesito de perfis, o trabalho do Plachevski [18] e Milke [21] possui um cadastro de usuário com características básicas de informações.

Por meio da análise dos trabalhos relacionados, pode-se perceber que dentre as características comparadas, apenas o TAADA tem disponibilidade de manuseio de configuração tal como o Hermes. Outro ponto que apresenta as mesmas características é o item perfil, encontrados nos aplicativos TAADA e na Tecnologia Assistiva para Pessoas com Deficiência Auditiva. Por essa razão, nota-se que o Hermes é o único a apresentar sensibilidade ao contexto, pois nenhum dos aplicativos comparados possibilita o reconhecimento de som, localização de recurso e por fim, suporte a trilhas.

\section{Conclusão}

Este artigo propôs um modelo de acessibilidade ubíqua voltada ao deficiente auditivo. O Hermes estende os recursos propostos pelo modelo Hefestos [3], o qual suporta diferentes padrões de acessibilidade.

Foram tomadas como base para a criação do protótipo a obtenção dos trabalhos relacionados, analisandose suas funcionalidades. Com base nas funcionalidades existentes, procurou-se desenvolver novas funções que atendessem de forma diária as necessidades dos deficientes auditivos, por isso, foi importante a busca por conhecer as tecnologias assistivas existentes para deficientes auditivos.

Ao realizar a avaliação de funcionalidade, verificou-se que o aplicativo funcionou de forma correta, desde a sua busca pelo local mais próximo onde o usuário pudesse conseguir o suporte de acessibilidade para obtenção de informação. Vale destacar que quando o usuário chegou a um contexto, o Hermes App enviou alerta, notificando a chegada dentro do recurso escolhido.

A avaliação desse cenário possibilitou a detecção de uma falha, quando, em um determinado momento, houver muitas falas de pessoas, ao passar-se próximo à usuária, o aplicativo não consegue identificar de forma correta as falas.

Entretanto, o aplicativo Hermes APP foi avaliado pelos participantes que aprovaram com $88 \%$ o aplicativo no quesito que buscou saber sobre a facilidade de uso, enquanto que $90 \%$ aprovaram quanto à utilidade percebida. De acordo com as pesquisas realizadas, em especial com foco nos trabalhos relacionados, percebe-se que as tecnologias assistivas voltadas para o atendimento dos deficientes auditivos desenvolvidas dentro do campo da computação ubíqua ainda são escassas. 
Os recursos existentes nas tecnologias existentes voltadas para surdos apresentaram a ausência de algumas funcionalidades que auxiliariam o surdo e facilitariam a sua acessibilidade. O aplicativo Hermes APP é uma proposta inicial que poderá ser aperfeiçoada. Durante os testes foi detectada uma falha na emissão da mensagem quando havia várias falas próximas ao dispositivo móvel, no momento em que o programa fazia reconhecimento de voz.

Por essa razão, destaca-se a exploração do melhoramento do reconhecimento de voz como o uso de palavras que serão padrões para esse reconhecimento de voz. Espera-se, no futuro, a exploração de sensores externos como pulseiras ou relógios vibratórios que possam chamar mais atenção ao deficiente auditivo e sensores externos de microfone mais apurados para o reconhecimento de voz.

Outras funcionalidades que poderão ser abordadas em trabalhos futuros são a inclusão de algoritmo para reconhecimento de som como uma sirene proposto por Mielke [21]. Funcionalidade importante para o Módulo de Eventos é a criação de um avatar que servirá para repassar ao deficiente auditivo os alertas na língua de sinais (Libras). Uma funcionalidade para o Módulo de Recursos é a opção de solicitar ajuda a perfis compatíveis com o tipo de acessibilidade por meio de localização por GPS.

\section{Agradecimentos}

Os autores agradecem à Coordenação de Núcleo de Inclusão do Instituto Federal de Ciência e Tecnologia de Roraima (IFRR) por ajudar a testar os cenários propostos pelo Hermes, envolvendo uma aluna que possui deficiência auditiva. Agradecem também aos participantes que realizaram o teste de usabilidade.

\section{Referências}

[1] IBGE, Instituto Brasileiro de Geografia e Estatística. Censo Demográfico 2000 (Tipo de Deficiência), $2000 . \quad$ Disponível em: $<$ http://www.ibge.gov.br/home/estatistica/populacao/censo2000/populacao/deficiencia_Censo2000.pdf>. Acesso em: 27 de fevereiro de 2016.

[2] IBGE, Instituto Brasileiro de Geografia e Estatística. Censo Demográfico 2010 (Tipo de Deficiência), 2010. Disponível em: $<$ http://www.ibge.gov.br/apps/snig/v1/?loc $=0 \&$ cat $=-1,-2,-3,128 \&$ ind $=4643>$. Acesso em: 27 de fevereiro de 2016.

[3] TAVARES, João E. R.; BARBOSA, Jorge L. V.; CARDOSO, Ismael G.; COSTA, Cristiano A; YAMIN, Adenauer C.; REAL, Rodrigo A. Hefestos: an intelligent system applied to ubiquitous accessibility. Universal Access in the Information Society (Print), v. 1, p. 1-20, 2015.

[4] VALMORBIDA, Willian; BARBOSA, Jorge L. V.; BARBOSA, Débora N. F. ; RIGO, Sandro J. . ULibrary: An Intelligent Model for Ubiquitous Library Support. Computer Journal (Print), v. 1, p. 1-20, 2015.

[5] SATYANARAYANAN, M. Pervasive Computing: vision and challenges. IEEE Personal Communications, v. 8, 2001. p. 10-17.

[6] DEY, A., SALBER, D., AND ABOWD G. 2001. A conceptual framework and a toolkit for supporting the rapid prototyping of context-aware application. Journal Human-Computer Interaction. v.16, 2, 97-166, 2001.

[7] WAGNER, André; BARBOSA, Jorge L. V.; BARBOSA, Débora N. F . A model for profile management applied to ubiquitous learning environments. Expert Systems with Applications, v. 41, p. 2023-2034, 2014.

[8] FALK, Roberto A.; TAVARES, João E. R.; BARBOSA, Jorge L.V. Tirésias: um modelo para acessibilidade ubíqua orientado à deficiência visual, Revista Brasileira de Computação Aplicada (ISSN 2176-6649), Passo Fundo, v. 5, n. 1, p. 55-70, abr. 2013 
[9] DEY, Anind K. Understanding and using context. Personal and Ubiquitous Computing, v. 5, n.1,p. 4-7, February 2001.

[10] PRIETCH, Soraia S.; SANTOS, Emanuel José dos; FILGUEIRAS, Lucia V. L. A Mean for Communication between Deaf and Hearing Pairs in Inclusive Educational Settings: The Sessai App. ACM. 2015. Disponível em: <http://dx.doi.org/10.1145/2745555.2746677>

[11] BRAGA, Newton C. Instituto Newton C. Braga. 2014. Disponível em: $<$ http://www.newtoncbraga.com.br/index.php/instrumentacao/108-artigos-diversos/3556-ins149>. Acesso em: 27 de fevereiro de 2016.

[12] MAFRA, Alexandre T. Reconhecimento Automático de Locutor em Modo Independente de Texto por SelfOrganizing Maps. Escola Politécnica da Universidade de São Paulo. 2005

[13] PADGHAM, L. AND WINIKOFF, M. Prometheus: A Methodology for Developing Intelligent Agents. Melbourne: [s.n.], 2002.

[14] SILVA, Jader M. et al. Content Distribution in Trail-aware Environments. Journal of the Brazilian Computer Society (Impresso), v. 16, p. 163-176, 2010.

[15] RAMIREZ, Daniel Hernandes. Measuring Decibels With Mobile Phone.2013. Disponível em: $<$ http://stackoverflow.com/questions/15693990/measuring-decibels-with-mobilephone?answertab=votes\#tab-top> Acesso em: 27 de fevereiro de 2016.

[16] DAVIS, F. Perceived usefulness, perceived ease of use, and user acceptance of information technology. MIS Q. 13(3), 319-340 (1989).

[17] PRODEAF. Disponível em: <http://www.prodeaf.net/ >. Acesso em: 27 de fevereiro de 2016.

[18] PLACHEVSKI, M. S. Sistema de Tecnologia Assistiva para Captar a Atenção de Deficientes Auditivos e Surdos. UNICAMP - Universidade Estadual de Campinas, 2014.

[19] HAND TALK. Disponível em: <http://www.handtalk.me/>. Acesso em: 27 de fevereiro de 2016.

[20] RYBENÁ. Disponível em: <http://www.rybena.com.br/site-rybena/>. Acesso em: 27 de fevereiro de 2016.

[21] MIELKE, Matthias; GRUNEWALD, Armin; BRUCK, Rainer. An Assistive Technology for HearingImpaired Persons: Analysis, Requirements and Architecture. 35th Annual International Conference of the IEEE EMBS, Osaka, Japan, p 4702-4705, 2013. 